\title{
Enhanced tunneling electroresistance in multiferroic tunnel junctions due to the reversible modulation of orbitals overlap
}

\author{
Leina Jiang, ${ }^{1,2}$ L. L. Tao, ${ }^{2}$ B. S. Yang, ${ }^{1}$ J. Wang, ${ }^{2, a)}$ and X. F. Han ${ }^{1, b)}$ \\ ${ }^{1}$ Beijing National Laboratory for Condensed Matter Physics, Institute of Physics, University of Chinese \\ Academy of Sciences, Chinese Academy of Sciences, Beijing 100190, China \\ ${ }^{2}$ Department of Physics and the Center of Theoretical and Computational Physics, The University of Hong \\ Kong, Hong Kong, China
}

(Received 11 September 2016; accepted 27 October 2016; published online 7 November 2016)

\begin{abstract}
We report a first-principles study of the ferroelectricity and spin-dependent transport through $\mathrm{Co} /$ $\mathrm{BaTiO}_{3} / \mathrm{CoO} / \mathrm{Co}$ multiferroic tunnel junctions (MFTJs). We find the coexistence of large tunneling magnetoresistance (TMR) ratio and large tunneling electroresistance (TER) ratio in the MFTJs. The large TMR effect originates from the spin-filter tunneling through the $\mathrm{BaTiO}_{3}$ barrier, while the TER effect is due to the modulation of orbitals overlap by polarization reversal. The microscopic physics of TER are identified and understood through the analysis of metal-oxygen relative displacements, local polarization magnitude, transmission in momentum space and real space scattering states. Our results provide a practical way to achieve the coexistence of large TER and TMR effects in MFTJs. Published by AIP Publishing. [http://dx.doi.org/10.1063/1.4967379]
\end{abstract}

The research of ferroelectric tunnel junctions (FTJs) has recently attracted great attention due to their rich physics and promising applications in electronic devices such as nonvolatile random access memories. ${ }^{1,2}$ A typical FTJ is made of two metallic electrodes sandwiching a thin ferroelectric (FE) film. ${ }^{2-5}$ The reversal of FE polarization may change the electron tunneling probability through the FE barrier, which yields the well-known tunneling electroresistance (TER) effect. ${ }^{1}$ It is commonly accepted that the asymmetry of FTJs plays a crucial role for the sizable TER. This asymmetry is mostly achieved either by using asymmetric electrodes ${ }^{6-12}$ or through interface engineering. ${ }^{13-19}$ For example, it has been shown in experiment that $\mathrm{La}_{0.7} \mathrm{Sr}_{0.3} \mathrm{MnO}_{3}$ (LSMO)/ $\mathrm{BaTiO}_{3}(\mathrm{BTO}) / \mathrm{Au}$ and $\mathrm{LSMO} / \mathrm{BTO} / \mathrm{Cu}$ asymmetric FTJs reveal sizable TER effect due to effective potential barrier modulation by the polarization reversal. ${ }^{11}$ The microscopic physics of TER effect in those asymmetric FTJs is due to the change in electrostatic potential induced by different screening lengths of the electrodes. ${ }^{6}$ Yin et al. ${ }^{16}$ observed a significant enhancement of TER $\left(\sim 10^{4} \%\right)$ by intercalating a thin doped manganite layer in the FTJs consisting of LSMO electrodes and BTO barrier due to phase transition at the interface induced by polarization switching. ${ }^{15}$ On the other hand, it is more intriguing to construct the multiferroic tunnel junctions (MFTJs) by using the ferromagnetic electrodes in FTJs. For a MFTJ, both the reversal of polarization orientation and the change of magnetic configurations of the two electrodes may change the tunneling resistance, which gives rise to four resistance states, as has been demonstrated in $\mathrm{SrRuO}_{3}(\mathrm{SRO}) /$ $\mathrm{BTO} / \mathrm{SRO}^{13}$ from first principles and reported in $\mathrm{Fe} / \mathrm{BTO} /$ $\mathrm{LSMO}^{20}$ and $\mathrm{Co} / \mathrm{PZT} / \mathrm{LSMO}^{21}$ experimentally.

The coexistence of large tunneling magnetoresistance (TMR) and TER effect is an important device merit of a MFTJ device. In this work, we propose an efficient way to enhance the TER effect, that is, modulating the orbitals overlap through the FE polarization. To illustrate our idea, we consider a prototypical $\mathrm{Co} / \mathrm{BTO} / \mathrm{CoO} / \mathrm{Co}$ MFTJ model, where the extra $\mathrm{CoO}$ layer at the interface can be introduced by oxidation process in experiment. We find the Ti-Ti bond lengths that determine the orbital overlaps strength are changed significantly upon the polarization reversal. As expected, the modulation of orbitals overlap yields a large transmission difference thereby resulting in a large TER effect. Moreover, the large TMR effect is also observed capitalizing on spin-filter tunneling through the BTO barrier.

The atomic and electronic structures were calculated using the Vienna ab-initio simulation package (VASP). ${ }^{22}$ We used an energy cutoff of $500 \mathrm{eV}$ and PBEsol generalized gradient approximation (GGA) for the exchange correlation functional. ${ }^{23}$ A $6 \times 6 \times 1 \mathrm{k}$-point sampling was used for atomic relaxations calculations until the forces on each atom were smaller than $0.01 \mathrm{eV} / \AA$. The atomic structure of the $\mathrm{Co} /$ BTO/CoO/Co MFTJ is shown in Fig. 1(a). The in-plane lattice constant $a$ is fixed as the calculated value of cubic $\mathrm{SrTiO}_{3}$ (STO), $a=3.906 \AA$ to simulate epitaxially grown on a STO substrate. Under such a constraint, the calculated lattice constants are $a=3.906 \AA, c=4.192 \AA$ (out-of-plane lattice constant) for bulk BTO and the lattice parameters for bulk Co are $a=2.762 \AA, c=2.788 \AA$, which is a slightly distorted bcc structure. The calculated polarization for the strained BTO is $49.3 \mu \mathrm{C} / \mathrm{cm}^{2}$, in reasonable agreement with previous results. $^{15,19}$

The quantum transport calculations are performed using the Nanodcal, ${ }^{24}$ which is based on the state-of-the-art technique by combining real-space density functional theory (DFT) with the Keldysh nonequilibrium Green's function (NEGF) formalism (NEGF-DFT). The spin-polarized conductance $G_{\sigma}$ is given by the Landauer-Büttiker formula,

$$
G_{\sigma}=\frac{e^{2}}{h} \sum_{\mathbf{k}_{\|}} T_{\sigma}\left(\mathbf{k}_{\|}, E_{F}\right),
$$

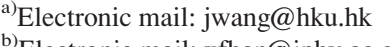

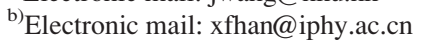



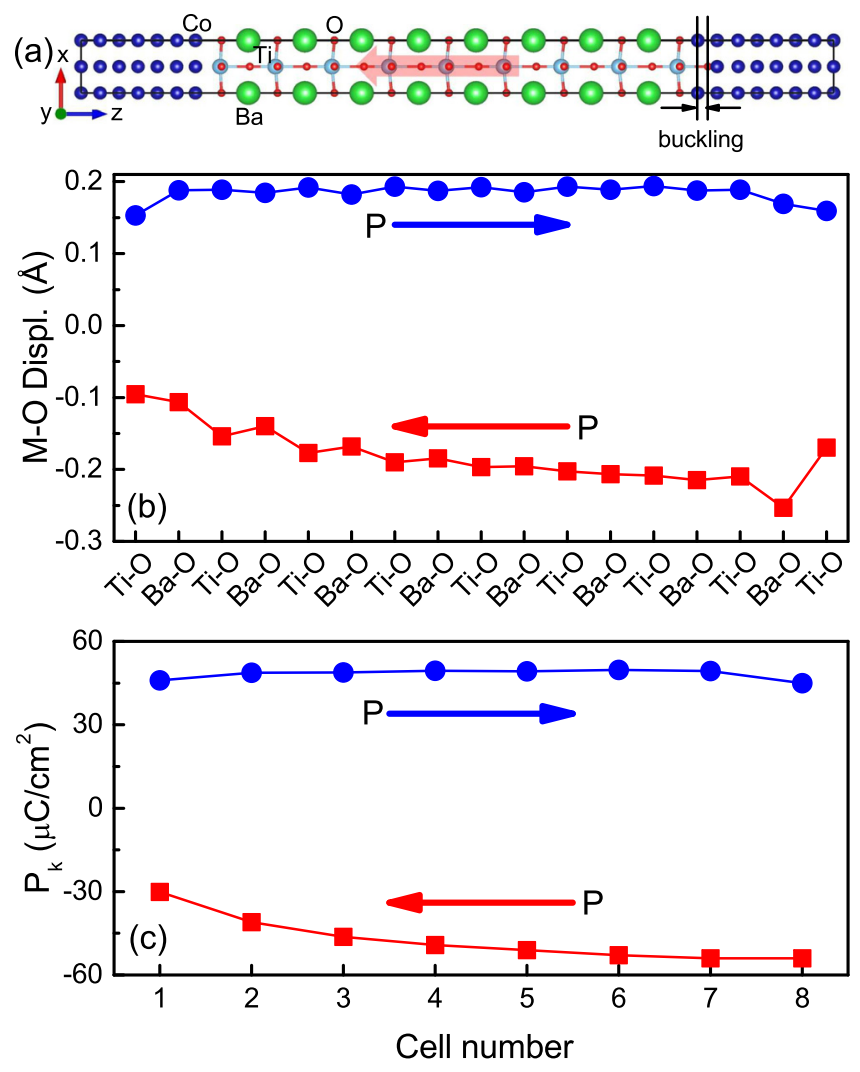

FIG. 1. (a) Fully relaxed atomic structure of the $\mathrm{Co} / \mathrm{BTO} / \mathrm{CoO} / \mathrm{Co}$ MFTJ with left polarization. The left interface termination is TiO-terminated type with $\mathrm{O}$ on the top of $\mathrm{Co}$, while $\mathrm{Co}$ and $\mathrm{O}$ of the right interfacial $\mathrm{CoO}$ layer are on hollow of $\mathrm{O}$ and on the top of $\mathrm{Ti}$, respectively. The interfacial buckling between $\mathrm{Co}$ and $\mathrm{O}$ is about $0.75 \AA(0.27 \AA)$ for left (right) polarization case. (b) The relative metal-O (M-O) displacements (Displ.) in the BTO layer for the left and the right polarization states as indicated by the arrows. (c) Local polarization $P$ mapped onto unit cell.

where $T_{\sigma}\left(\mathbf{k}_{\|}, E_{F}\right)$ is the transmission coefficient at the Fermi level $E_{F}$ with spin $\sigma(\sigma=\uparrow, \downarrow)$ and transverse Bloch wave vector $\mathbf{k}_{\|}=\left(k_{x}, k_{y}\right), e$ is the electron charge, and $h$ is the Planck's constant. A $10 \times 10 \mathbf{k}_{\|}$mesh for self-consistent calculations and a $100 \times 100 \mathbf{k}_{\|}$mesh for evaluating conductance are used. The generalized gradient approximation as parameterized by Perdew, Burke, and Ernzerhof is used for the exchange-correlation potential. ${ }^{25}$

First, we will examine the atomic structures and FE properties of the MFTJ. Fig. 1(b) shows relaxed relative metal-O (M-O) displacements in the BTO layer for either left $(\leftarrow$, negative displacements) or right $(\rightarrow$, positive displacements) polarization state. As expected, both the left and right FE polarization states are obtained as evident from the displacements. In addition, the displacements are significantly asymmetric that the displacements for right polarization are relatively uniform while the displacements for left polarization increase monotonously from left interface to right one except a sudden reduction for the most right layer. As we shall see later, the observed asymmetric displacements play a key role for the large TER effect. From those M-O displacements and the Born effective charges, the local polarization mapped on to the $k$ th unit cell $P_{k}$ can be evaluated by ${ }^{26}$

$$
P_{k}=\frac{1}{\Omega_{k}} \sum_{m} Z_{m}^{*} \delta z_{m}
$$

where $\Omega_{k}$ is the volume of the $k$ th unit cell, $Z_{m}^{*}$ is the Born effective charge, and $\delta z_{m}$ is the displacement of the $m$ th atom. $Z_{m}^{*}$ calculated using density functional perturbation theory ${ }^{27}$ for $\mathrm{Ba}, \mathrm{Ti}, \mathrm{O}_{\perp}(\mathrm{TiO}$ plane $)$ and $\mathrm{O} \|(\mathrm{BaO}$ plane $)$ ions are $2.70,6.67,-2.08$, and -5.21 electrons, respectively. $P_{k}$ is shown in Fig. 1(c). The magnitude of local polarization is in line with M-O displacements that the asymmetric local polarizations can be clearly seen. As such, the potential profiles are rather different between left and right polarization cases, which results in the expected TER effect.

To understand the above asymmetric M-O displacements, in Fig. 2 we plot the electrostatic potential profile through the MFTJ for artificial paraelectric (PE) state, namely, there are no relatively $\mathrm{M}-\mathrm{O}$ displacements and the interface distances are determined by the total energy minimization. We mention that such PE state can eliminate the effect of depolarizing field induced by the M-O displacements. A potential drop within BTO layer from the left interface to the right one can be seen clearly, indicating an intrinsic electric field $E_{i}$ pointing from $\mathrm{Co} / \mathrm{TiO}$ interface to $\mathrm{TiO} / \mathrm{CoO}$ interface. Thus, $E_{i}$ tends to enforce M-O displacements positive, that is, polarization pointing to the right. The magnitudes of $\mathrm{M}-\mathrm{O}$ displacements for right polarization are expected to be larger than those for left polarization. This is in line with the calculated results shown in Fig. 1(b) especially around the left interface.

We now investigate the TMR and TER effects, which are key parameters characterizing the performance of MFTJ devices. We define the TMR ratio as $T M R=\left(G_{P}-G_{A P}\right) / G_{A P}$, with $G_{P}$ and $G_{A P}$ being the total conductance for the MFTJ in parallel (PC) and anti-parallel configurations (AP), respectively. In a similar way, the TER ratio can be defined as $T E R=\left(G_{\leftarrow}-G_{\rightarrow}\right) / G_{\rightarrow}$, with $G_{\leftarrow}\left(G_{\rightarrow}\right)$ being the total conductance for the MFTJ in left (right) polarization. Note that the TER ratio now depends on the magnetic configurations of the two electrodes and vice versa; hence, we can obtain four resistance states in a single MFTJ element. All the relevant results are reported in Table I. For the left polarization case, we see that $G_{P}$ is more than ten times as large as $G_{A P}$ giving rise to large TMR ratio of more than $10^{3} \%$. TMR effect is

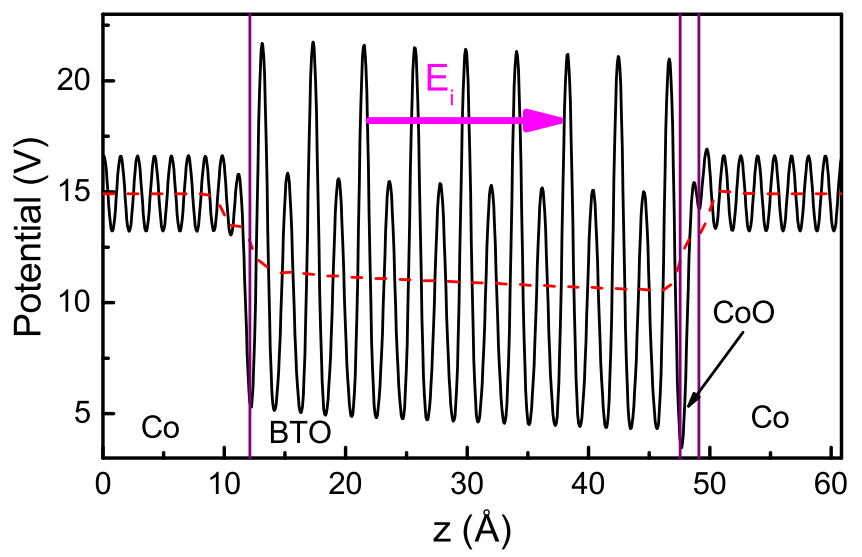

FIG. 2. Planar (black solid lines) and macroscopic averaged (red dashed lines) electrostatic potential along the $z$ direction for the MFTJs in a paraelectric $(\mathrm{PE})$ state. The vertical solid lines denote roughly the positions of interfaces. $E_{i}$ is the intrinsic electric field pointing from $\mathrm{Co} / \mathrm{TiO}$ interface to $\mathrm{TiO} / \mathrm{CoO}$ interface. 
TABLE I. Spin-polarized conductance $G_{\sigma}$ (in units of $10^{-8} e^{2} / h$ ), TMR and TER ratios (in \%) for the MFTJ in the left $(\leftarrow)$ polarization and the right $(\rightarrow)$ polarization states. $G_{\uparrow \uparrow}, G_{\downarrow \downarrow}$ are the majority-spin and minority-spin conductance in PC, respectively. $G_{\uparrow \downarrow}, G_{\downarrow \uparrow}$ are the majority-to-minority and minority-to-majority conductance in $\mathrm{AP}$, respectively.

\begin{tabular}{|c|c|c|c|c|c|}
\hline & \multicolumn{2}{|c|}{$P_{\leftarrow}$} & \multicolumn{2}{|c|}{$P_{\rightarrow}$} & \multirow[b]{2}{*}{$\operatorname{TER}(\%)$} \\
\hline & $G_{\uparrow \uparrow}$ & $G_{\downarrow \downarrow}$ & $G_{\uparrow \downarrow}$ & $G_{\downarrow \uparrow}$ & \\
\hline $\mathrm{PC}$ & 0.34 & 7.23 & 0.03 & 0.23 & 2812 \\
\hline $\mathrm{AP}$ & 0.07 & 0.44 & 0.07 & 0.09 & 219 \\
\hline TMR (\%) & \multicolumn{2}{|c|}{1384} & \multicolumn{2}{|c|}{63} & $\ldots$ \\
\hline
\end{tabular}

significantly reduced to $60 \%$ upon the polarization reversal from left to right. On the other hand, a large TER effect of more than $2000 \%$ is observed for the MFTJ in PC, and it is significantly reduced to $200 \%$ upon changing the magnetic configurations from $\mathrm{PC}$ to AP.

To understand the conductance change yielding the TMR or TER effect, according to Eq. (1), we plot the distribution of transmission coefficients in the two-dimensional Brillouin zone (BZ) in Fig. 3. From Figs. 3(a) and 3(b), we see that the transmission is mainly centered around the BZ center accompanied by large transmission along the $\Gamma-M$ direction. This arises from the slow decay through the $\Delta_{1}$ (spin up) and $\Delta_{5}$ (spin down) states, which can be confirmed from the symmetry-resolved band structures of bulk Co as shown in Fig. 4 and the complex band structure of BTO. ${ }^{28}$ We see that $\Delta_{1}$ band crosses the Fermi level only for spin up while $\Delta_{5}$ band crosses the Fermi level only for spin down, indicating that Co reveals half-metal nature in terms of both $\Delta_{1}$ and $\Delta_{5}$ symmetry states. The transmission coefficients are expected to be significantly reduced for the MFTJ in AP, as evident by comparing Fig. 3(c) with Fig. 3(a) or Fig. 3(d) with Fig. 3(b). The analysis is very similar to the right polarization case. The TMR mechanism is very similar to that predicted in the Co/STO/Co magnetic tunnel junctions ${ }^{29}$ due to the same symmetry for the complex band structure between BTO and STO. ${ }^{19}$ We conclude that the large TMR effect reported in Table I originates from the $\Delta_{1}$ and $\Delta_{5}$ spinfiltering effect, which is similar to $\Delta_{1}$ spin-filtering tunneling through $\mathrm{MgO}$ barrier. $^{30}$

We now analyze the physical origin of large TER effect. Here, we take the PC case as an example. Comparing Fig. 3(a)
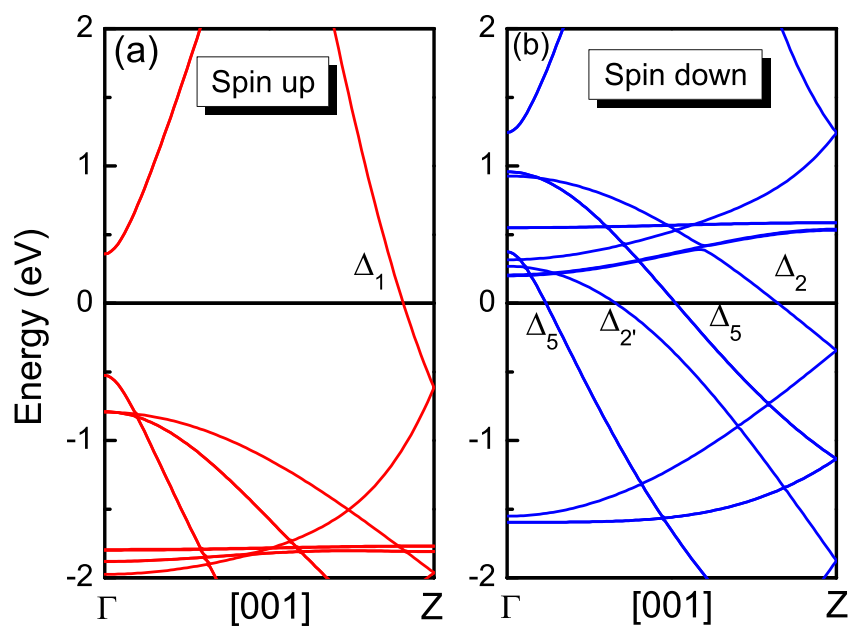

FIG. 4. (a) Spin up and (b) spin down band structures of bulk tetragonal Co along $\Gamma-Z$ direction. The Fermi level has been aligned to zero. The symmetries for the bands crossing the Fermi level are denoted.

with Fig. 3(e) or Fig. 3(b) with Fig. 3(f), it is clear that the transmission for the right polarization case is strongly depressed, which in turn yielding a large TER effect reported in Table I. To understand the observed TER effect, we plot the real-space scattering states through the MFTJ in Figs. 5(c)-5(h). Scattering states $\psi_{s}$ are eigenstates of the open twoterminal device structure linking $z=-\infty$ to $z=+\infty$, ${ }^{24}$ which are quite intuitive to analyze transport properties. Scattering states $\psi_{s}$, for example, going from left to right can be expressed as

$$
\psi^{k_{L}^{n}}=\left\{\begin{array}{l}
\phi^{k_{L}^{n}}+\sum_{m} \varphi^{k_{L}^{m}} r^{k_{L}^{m} k_{L}^{n}} \\
\psi_{C}^{k_{L}^{n}} \\
\sum_{m} \varphi^{k_{R}^{m}} t^{k_{R}^{m} k_{L}^{n}},
\end{array}\right.
$$

where $r, t$ are the reflection and transmission amplitudes, respectively. The superscript $m$ or $n$ labels the asymptotic Bloch wave vector $k^{m}$ or $k^{n}$ in the electrodes and $\phi, \varphi$ are incoming and outgoing states, respectively. Based on the converged open device Hamiltonian, one can obtain reflection and transmission amplitudes and then also the scattering states $\psi_{s}$ by matrix techniques. Figs. 5(c)-5(f) show the decay of planar averaged scattering states $\left|\psi_{s}(z)\right|$ (modulus) for different channels at the $\mathbf{k}_{\|}=(0,0)$ point through the
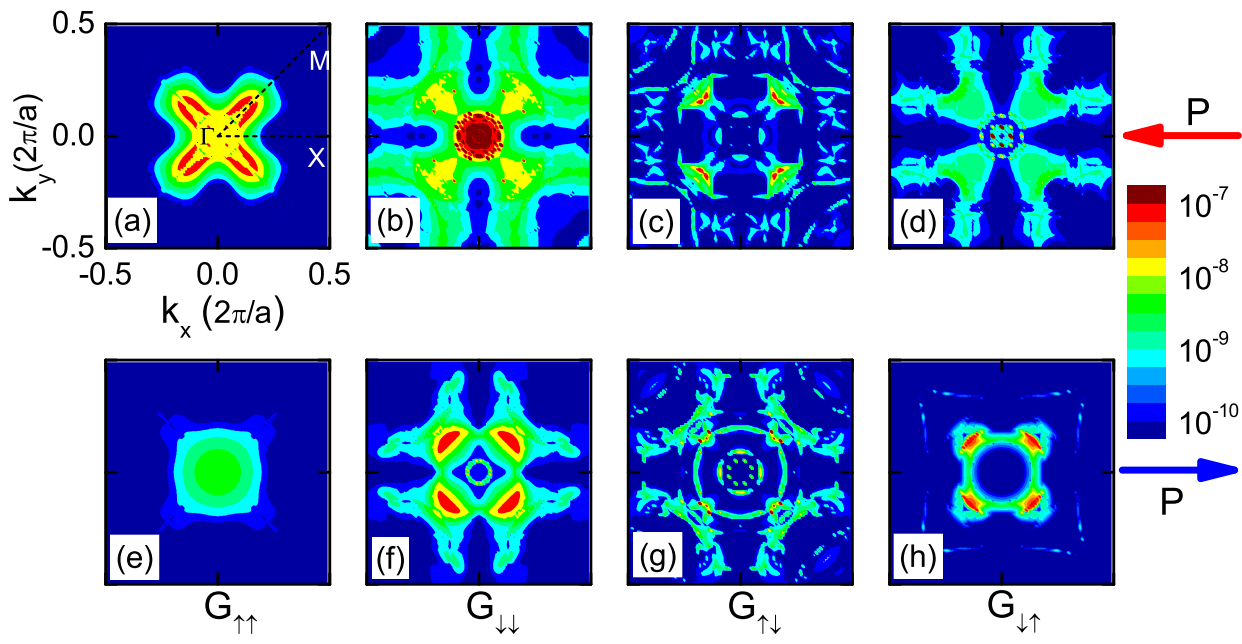

FIG. 3. (a) The spin and $k_{\|}$-resolved transmission coefficients at the Fermi energy in the two-dimensional Brillouin zone. Panels from the left to right are (a, e) majority-to-majority and (b, f) minority-to-minority in $\mathrm{PC}$; $(\mathrm{c}, \mathrm{g})$ for majority-to-minority and $(\mathrm{d}, \mathrm{h})$ for minority-to-majority in AP. The top panels (a-d) are for left polarization case while the bottom panels (e-h) are for right polarization case, as indicated by the arrows.
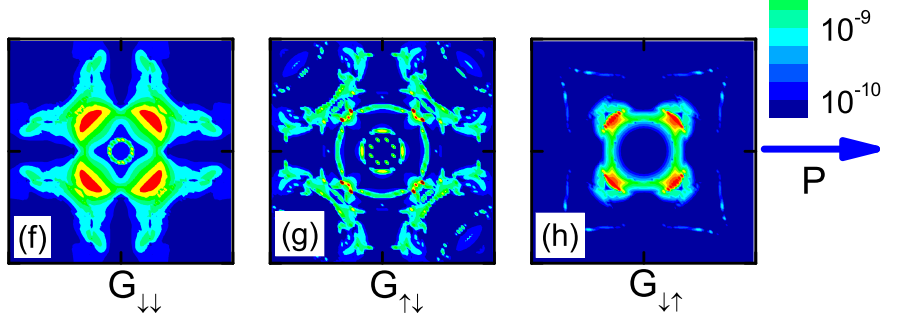


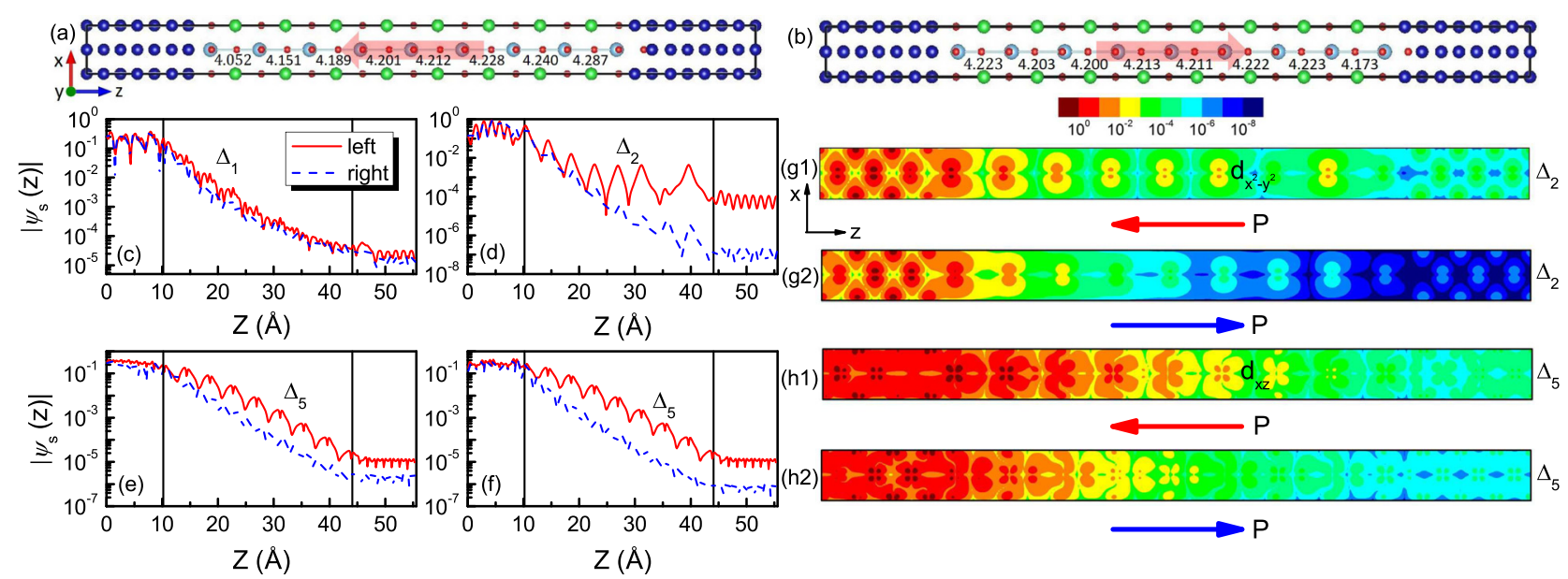

FIG. 5. Fully relaxed atomic structure of MFTJs with either (a) the left or (b) the right polarization as indicated by the red arrows. The numbers represent the Ti-Ti bond lengths (unit: $\AA$ ). (c, d, e, f) The distribution of real-space scattering states $\left|\psi_{s}(z)\right|$ (modulus) at $\mathbf{k}_{\|}=(0,0)$ point through the junction for left (red solid lines) and right (blue dashed lines) polarization states. $\left|\psi_{s}(z)\right|$ was averaged in the $x-y$ plane. The two vertical solid lines denote roughly the positions of interfaces. (c) Spin up $\Delta_{1}$ symmetry state, (d) spin down $\Delta_{2}$ symmetry state, and (e) and (f) spin down degenerate $\Delta_{5}$ symmetry states. (g, h) The distribution of real-space scattering states $\left|\psi_{s}(x, z)\right|$ at $\mathbf{k}_{\|}=(0,0)$ point. Spin down $\Delta_{2}$ symmetry state for (g1) the left and (g2) the right polarization states. Spin down $\Delta_{5}$ symmetry state for (h1) the left and (h2) the right polarization states. The arrows denote the polarization orientations.

junction. Overall, $\left|\psi_{s}(z)\right|$ decays exponentially through the barrier and decays much faster in right polarization case in compared with that of left polarization case. The difference in the magnitude of $\left|\psi_{s}(z)\right|$ after tunneling through the barrier can be seen clearly. In particular, the difference is significantly for the spin down $\Delta_{2}$ and $\Delta_{5}$ states. To gain further insight, we plotted the $\left|\psi_{s}(x, z)\right|$ in the (020) plane of MFTJ in Figs. 5(g) and 5(h). From Fig. 5(g1), we see that the Ti$d_{x^{2}-y^{2}}$ orbitals bond efficiently and form the effective transmission channel for the left polarization case. On the contrary, the overlap between Ti- $d_{x^{2}-y^{2}}$ orbitals is strongly reduced for the right polarization as seen from Fig. 5(g2). Such modulation of Ti- $d$ orbitals overlap by polarization reversal can be simply confirmed from the Ti-Ti bond lengths. Figs. 5(a) and 5(b) show the fully relaxed atomic structures for the MFTJs with either the left $\left(\mathrm{P}_{\leftarrow}\right)$ or the right $\left(\mathrm{P}_{\rightarrow}\right)$ polarization case and the numbers denote the Ti-Ti bond lengths. We see that the Ti-Ti bond length increases monotonously from the left interface to the right one for $\mathrm{P}_{\leftarrow}$. In contrast, the Ti-Ti bond length is uniform through the BTO layer for $\mathrm{P}_{\rightarrow}$. It is clear that the Ti-Ti bond lengths around the left interface for $\mathrm{P}_{\leftarrow}$ are smaller than those for $\mathrm{P}_{\rightarrow}$. Such difference in Ti-Ti bond lengths will result in different orbital overlap magnitudes, which yields the larger transmission for left polarization. The above analysis is also applicable to the $\Delta_{5}$ symmetry state in Fig. 5(h). We conclude that the large TER effect arises from the modulation of Ti- $d$ orbitals overlap by polarization.

We mention that the proposed TER mechanism due to the reversible modulation of orbitals overlap by polarization can also be understood from another viewpoint. For example, the c constants (Ti-Ti bond lengths) of BTO layer near the left interface for right polarization are larger than those for left polarization. The local band gap and decay rate of bulk BTO with the c constants determined by Ti-Ti bond lengths are therefore reduced for left polarization when compared with those for the right polarization, ${ }^{13}$ which in turn resulting in different tunneling conductance. Since the FE displacements for left polarization case are rather inhomogeneous, it is somewhat less straightforward to determine the complex band of BTO layer as a whole. The analysis from real-space scattering states should be more intuitive and appropriate in this work.

In summary, based on first-principles calculations, we have investigated the ferroelectricity and spin-dependent transport in $\mathrm{Co} / \mathrm{BTO} / \mathrm{CoO} / \mathrm{Co}$ MFTJ. The coexistence of large TMR and TER effect is predicted. The large TMR effect is due to the $\Delta_{1} / \Delta_{5}$ spin-filtering through BTO barrier while the large TER effect arises from the reversal modulation of Ti- $d$ orbitals overlap. Our results provide some guidelines for designing multifunctional spintronic devices, e.g., four-state memory and logic devices.

This work was supported by the 863 Plan Project of Ministry of Science and Technology [MOST, No. 2014AA032904], the MOST National Key Scientific Instrument and Equipment Development Projects [Grant No. 2011YQ120053], the National Natural Science Foundation of China [NSFC, Grant Nos. 11434014, 51229101, and 51620105004], the Strategic Priority Research Program (B) of the Chinese Academy of Sciences (CAS) [Grant No. XDB07030200], and the user fund of Wuhan National High Magnetic Field Center [PHMFF2015011]. This work was also supported by University Grant Council of Hong Kong (Contract No. AoE/P-04/08). The atomic structure visualisation was produced with the VESTA software. ${ }^{31} \mathrm{We}$ are grateful to the National Supercomputer Center in Tianjin for providing the computational facility.

${ }^{1}$ V. Garcia and M. Bibes, Nat. Commun. 5, 4289 (2014).

${ }^{2}$ E. Y. Tsymbal and H. Kohlstedt, Science 313, 181 (2006).

${ }^{3}$ T. Tybell, C. H. Ahn, and J.-M. Triscone, Appl. Phys. Lett. 75, 856 (1999).

${ }^{4}$ D. D. Fong, G. B. Stephenson, S. K. Streiffer, J. A. Eastman, O. Auciello, P. H. Fuoss, and C. Thompson, Science 304, 1650 (2004).

${ }^{5}$ V. Garcia, S. Fusil, K. Bouzehouane, S. Enouz-Vedrenne, N. D. Mathur, A. Barthelemy, and M. Bibes, Nature 460, 81 (2009). 
${ }^{6}$ M. Ye. Zhuravlev, R. F. Sabirianov, S. S. Jaswal, and E. Y. Tsymbal, Phys. Rev. Lett. 94, 246802 (2005).

${ }^{7}$ H. Kohlstedt, N. A. Pertsev, J. R. Contreras, and R. Waser, Phys. Rev. B 72, 125341 (2005).

${ }^{8}$ A. Zenkevich, M. Minnekaev, Yu. Matveyev, Yu. Lebedinskii, K. Bulakh, A. Chouprik, A. Baturin, K. Maksimova, S. Thiess, and W. Drube, Appl. Phys. Lett. 102, 062907 (2013).

${ }^{9}$ Z. Wen, C. Li, D. Wu, A. Li, and N. B. Ming, Nat. Mater. 12, 617 (2013).

${ }^{10}$ X. Liu, J. D. Burton, and E. Y. Tsymbal, Phys. Rev. Lett. 116, 197602 (2016).

${ }^{11}$ R. Soni, A. Petraru, P. Meuffels, O. Vavra, M. Ziegler, S. K. Kim, D. S. Jeong, N. A. Pertsev, and H. Kohlstedt, Nat. Commun. 5, 5414 (2014).

${ }^{12}$ L. L. Tao and J. Wang, J. Appl. Phys. 119, 224104 (2016).

${ }^{13}$ J. P. Velev, C. G. Duan, J. D. Burton, A. Smogunov, M. K. Niranjan, E. Tosatti, S. S. Jaswal, and E. Y. Tsymbal, Nano Lett. 9, 427 (2009).

${ }^{14}$ M. Y. Zhuravlev, Y. Wang, S. Maekawa, and E. Y. Tsymbal, Appl. Phys. Lett. 95, 052902 (2009).

${ }^{15}$ J. D. Burton and E. Y. Tsymbal, Phys. Rev. Lett. 106, 157203 (2011).

${ }^{16}$ Y. W. Yin, J. D. Burton, Y.-M. Kim, A. Y. Borisevich, S. J. Pennycook, S. M. Yang, T. W. Noh, A. Gruverman, X. G. Li, E. Y. Tsymbal, and Q. Li, Nat. Mater. 12, 397 (2013).

${ }^{17}$ L. L. Tao and J. Wang, Appl. Phys. Lett. 108, 062903 (2016).

${ }^{18}$ A. Tsurumaki-Fukuchi, H. Yamada, and A. Sawa, Appl. Phys. Lett. 103, 152903 (2013).
${ }^{19}$ N. M. Caffrey, T. Archer, I. Rungger, and S. Sanvito, Phys. Rev. Lett. 109, 226803 (2012).

${ }^{20}$ V. Garcia, M. Bibes, L. Bocher, S. Valencia, F. Kronast, A. Crassous, X. Moya, S. Enouz-Vedrenne, A. Gloter, D. Imhoff et al., Science 327, 1106 (2010).

${ }^{21}$ D. Pantel, S. Goetze, D. Hesse, and M. Alexe, Nat. Mater. 11, 289 (2012).

${ }^{22}$ G. Kresse and J. Furthmüller, Phys. Rev. B 54, 11169 (1996).

${ }^{23}$ J. P. Perdew, A. Ruzsinszky, G. I. Csonka, O. A. Vydrov, G. E. Scuseria, L. A. Constantin, X. Zhou, and K. Burke, Phys. Rev. Lett. 100, 136406 (2008).

${ }^{24}$ J. Taylor, H. Guo, and J. Wang, Phys. Rev. B 63, 245407 (2001); See http://nanoacademic.ca/ NanoAcademic Technologies, Inc., for the Nanodcal transport package.

${ }^{25}$ J. P. Perdew, K. Burke, and M. Ernzerhof, Phys. Rev. Lett. 77, 3865 (1996).

${ }^{26}$ B. Meyer and D. Vanderbilt, Phys. Rev. B 65, 104111 (2002).

${ }^{27}$ M. Gajdoš, K. Hummer, G. Kresse, J. Furthmüller, and F. Bechstedt, Phys. Rev. B 73, 045112 (2006).

${ }^{28}$ J. P. Velev, C. G. Duan, K. D. Belashchenko, S. S. Jaswal, and E. Y. Tsymbal, Phys. Rev. Lett. 98, 137201 (2007).

${ }^{29}$ J. P. Velev, K. D. Belashchenko, D. A. Stewart, M. van Schilfgaarde, S. S. Jaswal, and E. Y. Tsymbal, Phys. Rev. Lett. 95, 216601 (2005).

${ }^{30}$ L. L. Tao, S. H. Liang, D. P. Liu, H. X. Wei, J. Wang, and X. F. Han, Appl. Phys. Lett. 104, 172406 (2014).

${ }^{31}$ K. Momma and F. Izumi, J. Appl. Crystallogr. 44, 1272 (2011). 